\title{
Impact of nonalcoholic fatty liver disease on the risk of metachronous colorectal neoplasia after polypectomy
}

\author{
Nam Hee Kim, Yoon Suk Jung, Jung Ho Park, Dong Il Park, and Chong Il Sohn
}

Division of Gastroenterology, Department of Internal Medicine, Kangbuk Samsung Hospital, Sungkyunkwan University School of Medicine, Seoul, Korea

Received: October 29, 2019

Revised : January 3, 2020

Accepted: January 9, 2020

\section{Correspondence to}

Yoon Suk Jung, Ph.D.

Division of Gastroenterology,

Department of Internal

Medicine, Kangbuk Samsung

Hospital, Sungkyunkwan

University School of Medicine, 29

Saemunan-ro, Jongno-gu, Seoul 03181, Korea

Tel: $+82-2-2001-8577$

Fax: +82-2-2001-2049

E-mail: ys810.jung@samsung.com

https://orcid.org/0000-0002-

$1963-7170$
Background/Aims: Metabolic syndrome has been reported to be a risk factor for metachronous colorectal neoplasia (CRN). However, the impact of nonalcoholic fatty liver disease (NAFLD), a hepatic manifestation of metabolic syndrome, on the development of metachronous CRN after polypectomy has been rarely examined. We evaluated the association between NAFLD and the development of metachronous CRN after polypectomy.

Methods: Asymptomatic subjects who underwent abdominal ultrasonography and endoscopic removal of $\geq 1$ adenomas at the index colonoscopy between 2010 and 2014, and had a follow-up surveillance colonoscopy until 2017 were analyzed. Results: Of 6,182 participants, 2,642 (42.7\%) had NAFLD at the time of the index colonoscopy. Patients with NAFLD had significantly higher cumulative incidence rates of metachronous overall CRN than those without NAFLD in both men $(19.4 \%$ vs. $18.2 \%$ at 3 years and $49.2 \%$ vs. $44.0 \%$ at 5 years; $p=0.001$ ) and women (18.7\% vs. $10.5 \%$ at 3 years and $56.1 \%$ vs. $29.8 \%$ at 5 years; $p<0.001$ ). Even after adjusting for confounders, NAFLD remained independently associated with an increased risk of metachronous overall CRN in both men (adjusted hazard ratio [HR], 1.17; 95\% confidence interval [CI], 1.06 to 1.29) and women (adjusted HR, 1.63; 95\% CI, 1.27 to 2.07). Additionally, NAFLD was an independent risk factor for metachronous advanced CRN (ACRN) in women (adjusted HR, 2.61; 95\% CI, 1.27 to 5.37).

Conclusions: NAFLD is related to an increased risk of metachronous CRN after polypectomy. Especially, women with NAFLD are at an increased risk of developing metachronous ACRN. Our results indicate a possible effect of NAFLD on the pathogenesis of CRN.

Keywords: Non-alcoholic fatty liver disease; Colorectal neoplasms; Recurrence

\section{INTRODUCTION}

Colorectal cancer (CRC) is the third most commonly diagnosed cancer and the fourth leading cause of cancer-related deaths worldwide [1]. Screening colonoscopy with polypectomy is the most efficient strategy to reduce the incidence of CRC and its associated mortality [2]. Pa- tients undergoing adenoma removal are recommended to undergo follow-up surveillance colonoscopy because of their higher risk of developing metachronous advanced adenomas or cancer [3-5]. The current guidelines on post-polypectomy surveillance stratify adenomas into two risk groups based on the baseline adenoma characteristics, and recommend follow-up surveillance 
colonoscopy every 5 to 10 years for patients with low-risk adenomas (1 to 2 tubular adenomas $<10 \mathrm{~mm}$ in size with low-grade dysplasia) and every 3 years for those with high-risk adenomas ( $\geq 3$ adenomas or advanced adenomas) [3-5]. Recently, in addition to the baseline adenoma characteristics, some clinical features, such as the presence of metabolic syndrome, have also been established as important risk factors for the development of metachronous colorectal neoplasia (CRN) [6,7]. Identifying the risk factors for metachronous CRN can provide a more detailed and tailored approach to post-polypectomy surveillance for each individual patient.

Nonalcoholic fatty liver disease (NAFLD), a hepatic manifestation of metabolic syndrome, encompasses a histological spectrum ranging from simple steatosis to nonalcoholic steatohepatitis, and may subsequently progress to liver cirrhosis and hepatocellular carcinoma (HCC) [8]. However, the clinical burden of NAFLD is not confined to the liver [9]. NAFLD is also an emerging risk factor for type 2 diabetes, cardiovascular disease, and extrahepatic cancers, particularly CRC $[10,11]$. Several previous studies have reported a significant positive association between NAFLD and the prevalence of CRC [12,13]. In recent meta-analyses, the prevalence of colorectal adenomas (precursors of CRC) was also higher in patients with NAFLD $[14,15]$. Furthermore, the severity of NAFLD has been reported to be significantly correlated with the risk of CRN $[16,17]$. The results of previous studies have suggested that patients with NAFLD may also have an increased risk of developing metachronous CRN after polypectomy. However, there is a lack of data on the association between NAFLD and the risk of metachronous CRN development. Therefore, we aimed to compare the risk of metachronous CRN according to the presence or absence of NAFLD, and to identify whether NAFLD is associated with the development of metachronous CRN after polypectomy.

\section{METHODS}

\section{Study population}

The Kangbuk Samsung Health Study is a cohort study including South Korean men and women (aged $\geq 18$ years) who underwent a comprehensive annual or biennial health examination at clinics associated with the
Kangbuk Samsung Hospital Total Healthcare Center in Seoul and Suwon, Korea. The study population comprised a subset of the Kangbuk Samsung Health Study subjects who underwent screening colonoscopy as part of a comprehensive health examination between 2010 and 2017. We retrospectively analyzed the data obtained from a prospectively established cohort.

Patients who underwent abdominal ultrasonography (US) and endoscopic removal of $\geq 1$ adenomas at the index colonoscopy between 2010 and 2014, and had a follow-up surveillance colonoscopy at an interval of $\geq 1$ year until 2017, were considered to fulfill the inclusion criteria. All polyps were removed endoscopically at the index colonoscopy, and a single follow-up colonoscopy was performed in all patients. On the same day as the index colonoscopy, abdominal US was performed before the colonoscopy procedure.

In South Korea, the Industrial Safety and Health Law mandates free annual or biennial health screening examinations of all employees. Most of the participants of our study were employees of various companies and local governmental organizations, or their spouses. As part of their welfare policy, companies often subsidize comprehensive health examinations, including abdominal US and colonoscopy. In this study, general practitioners interviewed all participants before colonoscopy to ensure that they were asymptomatic (i.e., no abdominal pain, diarrhea, or hematochezia). Symptomatic participants were urged to seek medical care.

The exclusion criteria were as follows: a history of CRC or colorectal surgery, a history of inflammatory bowel disease, a diagnosis of CRC at the index colonoscopy, $>10$ colorectal adenomas at the index colonoscopy, $\leq 1$ year interval before the follow-up surveillance colonoscopy, age < 30 years, poor bowel preparation at the index or follow-up colonoscopy, excessive alcohol consumption (> 140 g/week for men and > $70 \mathrm{~g} /$ week for women), positivity to hepatitis B virus surface antigen, positivity to hepatitis $\mathrm{C}$ virus antibody, and a history of HCC. Poor bowel preparation was defined as "large amounts of solid fecal matter observed in the bowel precluding a satisfactory study, unacceptable preparation, or a condition in which $<90 \%$ of the mucosa could be adequately visualized" [18].

This study was approved by the Institutional Review Board of Kangbuk Samsung Hospital (approval no.2017- 
11-011, approved on November 7, 2017). The requirement for informed consent was waived because only de-identified data were retrospectively assessed.

\section{Colonoscopic and histological examinations}

Board-certified endoscopists performed all colonoscopic examinations using an EVIS LUCERA CV-260 colonoscope (Olympus Medical Systems, Tokyo, Japan). All detected polyps were removed through biopsy, polypectomy, or endoscopic mucosal resection, and the specimens were histologically assessed by experienced pathologists.

Overall CRN was defined as a cancer or any adenoma, and advanced CRN (ACRN) was defined as a cancer or advanced adenoma. Advanced adenoma was defined as the presence of one of the following features: $\geq 10 \mathrm{~mm}$ diameter, tubulovillous or villous structure, and highgrade dysplasia [3].

\section{Measurements and definitions of variables}

Data on health-related behaviors and medical history were obtained through a self-administered questionnaire, whereas physical measurements were obtained by trained staff. Smoking status was categorized as never, former, or current smoking. Family history of CRC was defined as the occurrence of CRC in $\geq 1$ first-degree relatives at any age. Self-reported use of nonsteroidal anti-inflammatory drugs (NSAIDs) regularly over the month before study enrollment was recorded. The weekly frequency of moderate or vigorous physical activity was also assessed, and regular exercise was defined as exercising $\geq 3$ times per week. Obesity was defined as a body mass index of $\geq 25 \mathrm{~kg} / \mathrm{m}^{2}$, which is the proposed cutoff value for diagnosing obesity in Asians [19].

The presence or absence of fatty liver was examined on abdominal US, which was performed using a 3.5MHz transducer (LOGIQ 9; General Electric, Madison, WI, USA) by experienced radiologists who were unaware of the study aims. An ultrasonographic diagnosis of fatty liver was defined as the presence of a diffuse increase in fine echoes in the liver parenchyma compared with the kidney or spleen parenchyma [20]. For the diagnosis of fatty liver, the intraobserver agreement was extremely high (kappa 0.94) and the interobserver agreement was not low (kappa, 0.74) [21,22]. NAFLD was defined as the presence of fatty liver on abdominal US without other causes of chronic liver diseases as those described in the exclusion criteria.

\section{Statistical analysis}

Data are expressed as mean \pm standard deviation or frequency (\%). Baseline characteristics according to the presence or absence of NAFLD were compared using the chi-square test and Student's t-test for categorical and continuous variables, respectively. The cumulative incidence of metachronous CRN according to the presence or absence of NAFLD was calculated using the Kaplan-Meier method, and the difference was determined using log-rank test. The hazard ratios (HRs) and corresponding $95 \%$ confidence intervals (CIs) for metachronous CRN were estimated using multivariable Cox proportional hazard regression analysis after adjusting for confounders including age, smoking status, family history of CRC, use of NSAIDs, obesity, regular exercise, and baseline adenoma characteristics. All reported $p$ values were 2 -tailed, and $p<0.05$ was considered statistically significant. The software program SPSS version 21 (IBM Corp., Armonk, NY, USA) was used for statistical analyses.

\section{RESULTS}

\section{Baseline characteristics of the study population}

A total of 12,377 patients who underwent abdominal US and endoscopic removal of $\geq 1$ adenomas at the time of the index colonoscopy, and had a follow-up surveillance colonoscopy, were eligible for inclusion in this study. Among them, 2,725 patients were excluded for the following reasons: a history of CRC or colorectal surgery $(n=97)$, a history of inflammatory bowel disease $(n=35)$, a diagnosis of CRC at the index colonoscopy $(n=9),>10$ colorectal adenomas at the index colonoscopy $(n=3)$, $\leq 1$ year interval before the follow-up colonoscopy ( $\mathrm{n}=$ $278)$, age $<30$ years $(\mathrm{n}=131)$, and poor bowel preparation $(n=2,172)$. Additionally, 3470 patients with other causes of chronic liver diseases were excluded for the following reasons: excessive alcohol consumption $(n=3,098)$, positivity to hepatitis B virus surface antigen $(n=363)$, positivity to hepatitis $\mathrm{C}$ virus antibody $(\mathrm{n}=8)$, and a history of HCC $(n=1)$. Finally, 6,182 patients were judged eligible for this study (Fig. 1). 
Table 1. Baseline characteristics according to nonalcoholic fatty liver disease status

\begin{tabular}{|c|c|c|c|c|}
\hline Characteristic & $\begin{array}{c}\text { Total } \\
(\mathrm{n}=6,182)\end{array}$ & $\begin{array}{l}\text { NAFLD (-) } \\
(\mathrm{n}=3,540)\end{array}$ & $\begin{array}{l}\text { NAFLD }(+) \\
(\mathrm{n}=2,642)\end{array}$ & $p$ value \\
\hline Men & 4,659 & 2,328 & 2,331 & \\
\hline Age, yr & $43 \cdot 3 \pm 8.2$ & $43.3 \pm 8.5$ & $43 \cdot 3 \pm 7 \cdot 9$ & 0.967 \\
\hline Current or former smoking, $\%$ & 63.7 & 62.0 & 65.5 & 0.015 \\
\hline Family history of CRC, \% & 6.3 & 6.3 & 6.3 & 0.991 \\
\hline Use of NSAIDs, \% & 3.2 & 3.0 & $3 \cdot 4$ & 0.364 \\
\hline BMI, $\mathrm{kg} / \mathrm{m}^{2}$ & $24.7 \pm 2.9$ & $23.4 \pm 2.4$ & $26.0 \pm 2.7$ & $<0.001$ \\
\hline Obesity, BMI $\geq 25 \mathrm{~kg} / \mathrm{m}^{2}, \%$ & $43 \cdot 3$ & 23.8 & 62.8 & $<0.001$ \\
\hline Regular exercise $^{\mathrm{a}}, \%$ & 13.7 & 15.8 & 11.6 & $<0.001$ \\
\hline Baseline adenoma characteristics & & & & 0.082 \\
\hline Low-risk adenoma, \% & 83.9 & $84 \cdot 9$ & 83.0 & \\
\hline High-risk adenoma, \% & 16.1 & 15.1 & 17.0 & \\
\hline Interval between index and follow-up colonoscopy, yr & $3.5 \pm 1.4$ & $3.5 \pm 1.4$ & $3.5 \pm 1.4$ & 0.762 \\
\hline Women & 1,523 & 1,212 & 311 & \\
\hline Age, yr & $44 \cdot 7 \pm 8.8$ & $43 \cdot 6 \pm 8.3$ & $48.9 \pm 9.4$ & $<0.001$ \\
\hline Current or former smoking, \% & $4 \cdot 5$ & $4 \cdot 4$ & $5 \cdot 1$ & 0.559 \\
\hline Family history of CRC, \% & 6.6 & 6.6 & 6.8 & 0.924 \\
\hline Use of NSAIDs, $\%$ & 4.8 & 4.7 & 5.1 & 0.745 \\
\hline BMI, $\mathrm{kg} / \mathrm{m}^{2}$ & $22.6 \pm 3.2$ & $21.8 \pm 2.5$ & $25 \cdot 9 \pm 3.5$ & $<0.001$ \\
\hline Obesity, BMI $\geq 25 \mathrm{~kg} / \mathrm{m}^{2}, \%$ & 18.7 & 9.0 & 56.6 & $<0.001$ \\
\hline Regular exercise $^{\mathrm{a}}, \%$ & $17 \cdot 9$ & 18.0 & $17 \cdot 2$ & 0.748 \\
\hline Baseline adenoma characteristics & & & & 0.114 \\
\hline Low-risk adenoma, \% & 83.1 & 83.8 & 80.1 & \\
\hline High-risk adenoma, \% & 16.9 & 16.2 & $19 \cdot 9$ & \\
\hline Interval between index and follow-up colonoscopy, yr & $3.4 \pm 1.4$ & $3.5 \pm 1.4$ & $3 \cdot 3 \pm 1.3$ & 0.027 \\
\hline
\end{tabular}

Values are presented as mean \pm standard deviation.

NAFLD, nonalcoholic fatty liver disease; CRC, colorectal cancer; NSAIDs, nonsteroidal anti-inflammatory drugs; BMI, body mass index.

${ }^{\mathrm{a}}$ Moderate or vigorous physical activity $\geq 3$ times per week.

Of the 6,182 eligible patients, 2,642 (42.7\%) had NAFLD at the time of the index colonoscopy. The mean age of the study participants was $43.6 \pm 8.4$ years, and the proportion of men was $75.4 \%$.

The baseline characteristics of the study participants are described in Table 1. Of the 4,659 male patients, 2331 (50.0\%) had NAFLD at the time of the index colonoscopy. Among the men, the proportion of smokers and patients with obesity was higher in the NAFLD group than in the non-NAFLD group, whereas the proportion of patients with regular exercise was lower in the NAFLD group than in the non-NAFLD group. The mean interval between the index and follow-up colonoscopy was $3.5 \pm 1.4$ years, and the intervals were not significantly different between the two groups.

Of the 1,523 women, 311 (20.4\%) had NAFLD at the time of index colonoscopy. Among the women, the mean age and the incidence of obesity were higher in patients with NAFLD than in those without NAFLD. The mean interval between the index and follow-up colonoscopy was $3.4 \pm 1.4$ years, and the intervals were longer in patients without NAFLD than in those with NAFLD. 


\section{Cumulative risk of metachronous CRN according to the presence or absence of NAFLD}

Overall CRN developed in 1,999 (32.3\%) patients and ACRN developed in 144 (2.3\%) patients during a median follow-up of 3.3 years. The cumulative incidence rates

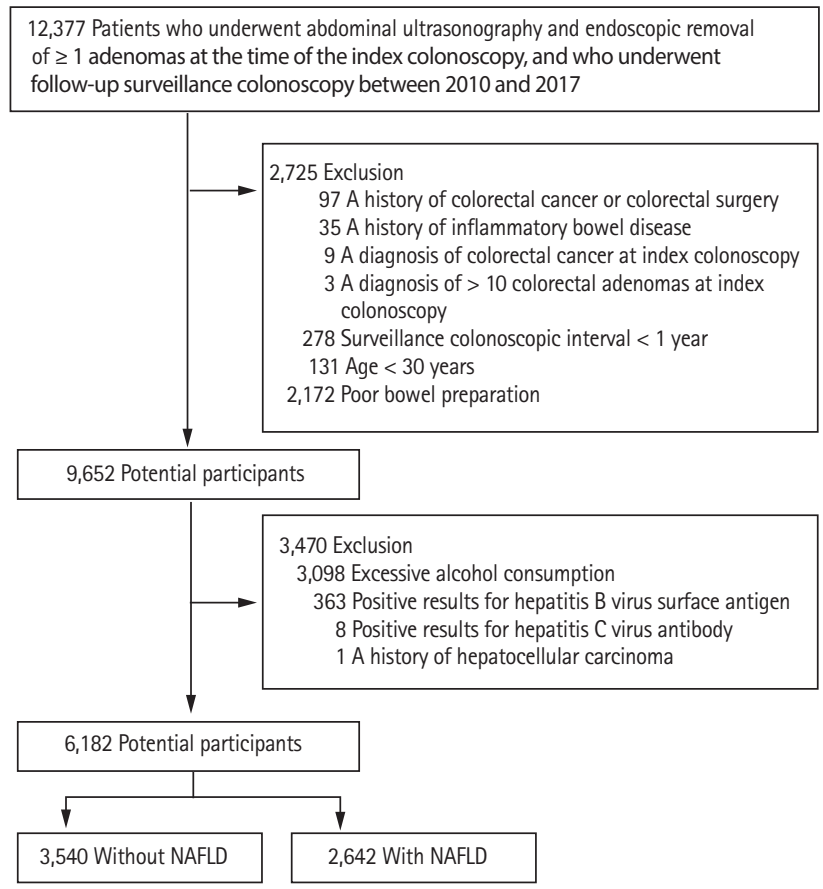

Figure 1. Flow diagram illustrating the selection of study subjects. NAFLD, nonalcoholic fatty liver disease.

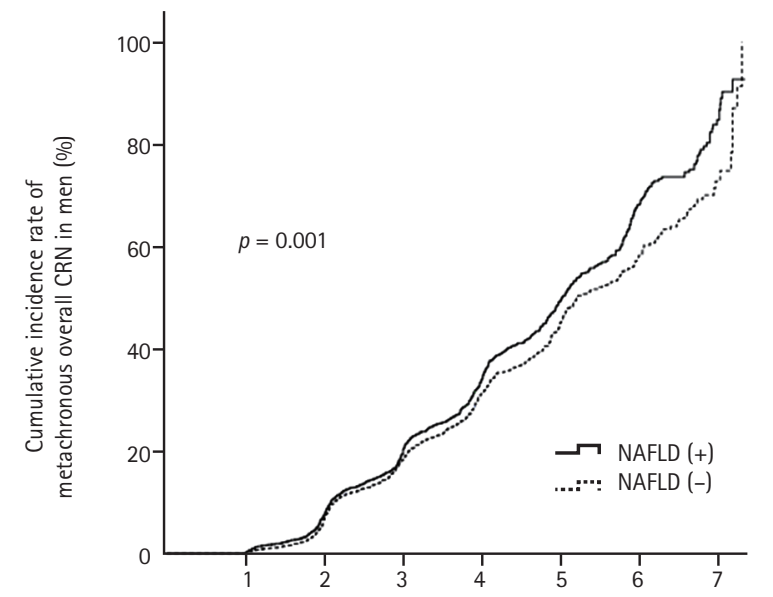

A

Follow-up period (yr) of metachronous overall CRN on follow-up colonoscopy were compared between patients with and without NAFLD, separately in men and women (Fig. 2). In men, the 3- and 5-year cumulative incidence rates of metachronous overall CRN were significantly higher in patients with NAFLD than in those without NAFLD (19.4\% vs. $18.2 \%$ and $49.2 \%$ vs. $44.0 \%$, respectively; log-rank $p=$ 0.001 ) (Fig. 2A). In women, the 3- and 5-year cumulative incidence rates of metachronous overall CRN were significantly higher in patients with NAFLD than in those without NAFLD ( $18.7 \%$ vs. $10.5 \%$ and $56.1 \%$ vs. $29.8 \%$, respectively; log-rank $p<0.001$ ) (Fig. 2B).

The 3- and 5-year cumulative incidence rates of metachronous ACRN were significantly higher in patients with NAFLD than in those without NAFLD among women (2.5\% vs. $0.8 \%$ and $10.3 \%$ vs. $2.9 \%$, respectively; log-rank $p=0.001$ ) (Fig. 3 B) but not among men (1.6\% vs. $1.4 \%$ and $3.7 \%$ vs. $4.0 \%$, respectively; log-rank $p=0.883$ ) (Fig. 3A).

\section{Risk factors associated with metachronous CRN}

Table 2 shows the results of univariable and multivariable Cox regression analyses for the factors associated with the development of metachronous overall CRN. In the univariable analysis, NAFLD was associated with an increased risk of metachronous overall CRN development in both men (HR, 1.18; 95\% CI, 1.07 to 1.29) and women (HR, 1.99; 95\% CI, 1.59 to 2.50). Even after adjust-

Figure 2. Cumulative incidence rate of metachronous overall colorectal neoplasia (CRN) according to the presence or absence of nonalcoholic fatty liver disease (NAFLD) in men (A) and women (B). 

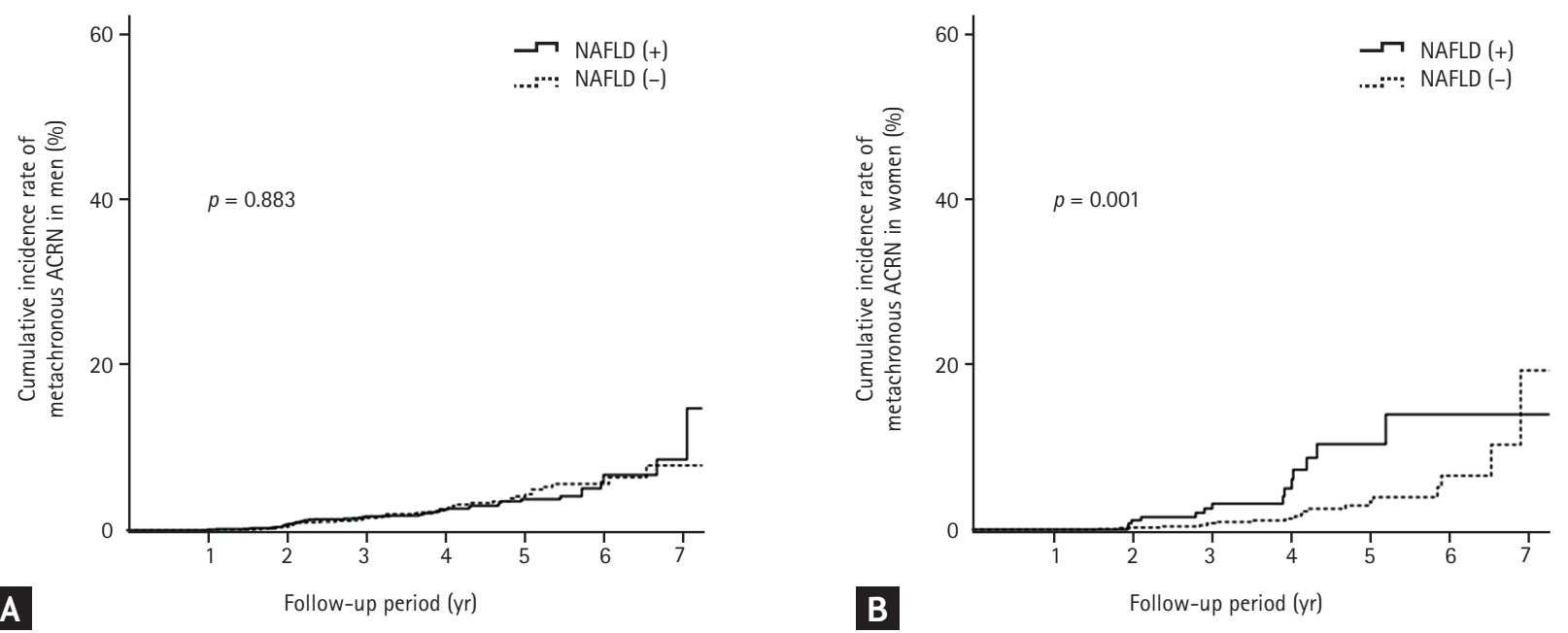

Figure 3. Cumulative incidence rate of metachronous advanced colorectal neoplasia (ACRN) according to the presence or absence of nonalcoholic fatty liver disease (NAFLD) in men (A) and women (B).

Table 2. Risk factors for metachronous overall colorectal neoplasia

\begin{tabular}{|c|c|c|c|c|}
\hline \multirow{2}{*}{ Variable } & \multicolumn{2}{|c|}{ Crude HR (95\% CI) } & \multicolumn{2}{|c|}{ Adjusted HR $(95 \% \text { CI })^{\mathrm{a}}$} \\
\hline & Men & Women & Men & Women \\
\hline \multicolumn{5}{|l|}{ NAFLD } \\
\hline No & 1 (reference) & 1 (reference) & 1 (reference) & 1 (reference) \\
\hline Yes & $1.18(1.07-1.29)$ & $1.99(1.59-2.50)$ & $1.17(1.06-1.29)$ & $1.63(1.27-2.07)$ \\
\hline Age, yr & $1.04(1.03-1.04)$ & $1.05(1.04-1.06)$ & $1.04(1.03-1.04)$ & $1.04(1.03-1.05)$ \\
\hline Current or former smoking & $1.08(0.98-1.19)$ & $0.92(0.56-1.51)$ & $1.06(0.96-1.18)$ & $0.88(0.53-1.45)$ \\
\hline Family history of CRC & $1.32(1.09-1.59)$ & $1.27(0.86-1.87)$ & $1.22(1.01-1.48)$ & $1.24(0.83-1.85)$ \\
\hline Use of NSAIDs & $1.10(0.85-1.42)$ & $0.89(0.54-1.47)$ & $1.04(0.80-1.35)$ & $0.87(0.52-1.46)$ \\
\hline Regular exercise ${ }^{\mathrm{b}}, \%$ & $1.03(0.90-1.18)$ & $1.07(0.80-1.43)$ & $0.94(0.81-1.08)$ & $0.99(0.74-1.33)$ \\
\hline \multicolumn{5}{|c|}{ Baseline adenoma characteristics } \\
\hline Low-risk adenoma & 1 (reference) & 1 (reference) & 1 (reference) & 1 (reference) \\
\hline High-risk adenoma & $1.57(1.40-1.77)$ & $1.58(1.21-2.05)$ & $1.40(1.24-1.59)$ & $1.28(0.97-1.68)$ \\
\hline
\end{tabular}

HR, hazard ratio; CI, confidence interval; NAFLD, nonalcoholic fatty liver disease; CRC, colorectal cancer; NSAID, nonsteroidal anti-inflammatory drug.

${ }^{a}$ Values were adjusted for NAFLD, age, current or former smoking, family history of CRC, use of NSAIDs, regular exercise, and baseline adenoma characteristics.

${ }^{\mathrm{b}}$ Moderate or vigorous physical activity $\geq 3$ times per week.

ing for potential confounders, NAFLD was still identified as an independent risk factor for metachronous overall CRN in both men (adjusted HR, 1.17; 95\% CI, 1.06 to 1.29 ) and women (adjusted HR, 1.63; 95\% CI, 1.27 to 2.07).

In addition, NAFLD was independently associated with an increased risk of metachronous ACRN in women (adjusted HR, 2.61; 95\% CI, 1.27 to 5.37). However, there was no significant association between NAFLD and the risk of metachronous ACRN in men (Table 3).

We further performed a subgroup analysis according to menopausal status (Table 4). Of the 1,523 female pa- 
Table 3. Risk factors for metachronous advanced colorectal neoplasia

\begin{tabular}{|c|c|c|c|c|}
\hline \multirow{2}{*}{ Variable } & \multicolumn{2}{|c|}{ Crude HR (95\% CI) } & \multicolumn{2}{|c|}{ Adjusted HR $(95 \% \mathrm{CI})^{\mathrm{a}}$} \\
\hline & Men & Women & Men & Women \\
\hline \multicolumn{5}{|l|}{ NAFLD } \\
\hline No & 1 (reference) & 1 (reference) & 1 (reference) & 1 (reference) \\
\hline Yes & $0.97(0.67-1.42)$ & $3.08(1.56-6.07)$ & $0.97(0.66-1.42)$ & $2.61(1.27-5.37)$ \\
\hline Age, yr & $1.05(1.03-1.07)$ & $1.06(1.03-1.10)$ & $1.04(1.02-1.67)$ & $1.04(1.01-1.08)$ \\
\hline Current or former smoking & $1.30(0.86-1.96)$ & $1.11(0.27-4.64)$ & $1.21(0.80-1.83)$ & $1.04(0.24-4.58)$ \\
\hline Family history of CRC & $0.82(0.33-2.01)$ & $0.44(0.06-3.20)$ & $0.76(0.31-1.88)$ & $0.51(0.07-3.72)$ \\
\hline Use of NSAIDs & $1.12(0.41-3.05)$ & $0.52(0.07-3.79)$ & $1.21(0.44-3.29)$ & $0.52(0.07-3.81)$ \\
\hline Regular exercise ${ }^{\mathrm{b}}, \%$ & $1.29(0.78-2.11)$ & $1.45(0.63-3.36)$ & $1.10(0.66-1.82)$ & $1.45(0.61-3.44)$ \\
\hline \multicolumn{5}{|c|}{ Baseline adenoma characteristics } \\
\hline Low-risk adenoma & 1 (reference) & 1 (reference) & 1 (reference) & 1 (reference) \\
\hline High-risk adenoma & $3.51(2.37-5.20)$ & $4.20(2.13-8.30)$ & $2.96(1.97-4.45)$ & $3.66(1.80-7.45)$ \\
\hline
\end{tabular}

HR, hazard ratio; CI, confidence interval; NAFLD, nonalcoholic fatty liver disease; CRC, colorectal cancer; NSAID, nonsteroidal anti-inflammatory drug.

${ }^{a}$ Values were adjusted for NAFLD, age, current or former smoking, family history of CRC, use of NSAIDs, regular exercise, and baseline adenoma characteristics.

${ }^{\mathrm{b}}$ Moderate or vigorous physical activity $\geq 3$ times per week.

Table 4. Subgroup analysis according to menopausal status

\begin{tabular}{|c|c|c|c|c|}
\hline \multirow[b]{2}{*}{ Variable } & \multicolumn{2}{|c|}{ Metachronous overall CRN } & \multicolumn{2}{|c|}{ Metachronous ACRN } \\
\hline & $\begin{array}{c}\text { Crude HR } \\
(95 \% \mathrm{CI})\end{array}$ & $\begin{array}{c}\text { Adjusted HR } \\
(95 \% \mathrm{CI})^{\mathrm{a}}\end{array}$ & $\begin{array}{c}\text { Crude HR } \\
(95 \% \mathrm{CI})\end{array}$ & $\begin{array}{c}\text { Adjusted HR } \\
(95 \% \mathrm{CI})^{\mathrm{a}}\end{array}$ \\
\hline \multicolumn{5}{|l|}{ Total women $(n=1,263)$} \\
\hline NAFLD $(-)(n=987)$ & 1 (reference) & 1 (reference) & 1 (reference) & 1 (reference) \\
\hline $\operatorname{NAFLD}(+)(\mathrm{n}=276)$ & $1.97\left(1.55^{-2.52)}\right.$ & $1.65(1.27-2.14)$ & $2.66(1.28-5.53)$ & $2.25(1.02-4.95)$ \\
\hline \multicolumn{5}{|c|}{ Premenopausal women $(\mathrm{n}=863)$} \\
\hline $\operatorname{NAFLD}(-)(\mathrm{n}=720)$ & 1 (reference) & 1 (reference) & 1 (reference) & 1 (reference) \\
\hline $\operatorname{NAFLD}(+)(\mathrm{n}=143)$ & $1.37(0.92-2.05)$ & $1.37(0.90-2.09)$ & $2.55(0.88-7.39)$ & $2.90(0.93-9.07)$ \\
\hline \multicolumn{5}{|c|}{ Postmenopausal women $(n=400)$} \\
\hline $\operatorname{NAFLD}(-)(\mathrm{n}=267)$ & 1 (reference) & 1 (reference) & 1 (reference) & 1 (reference) \\
\hline $\operatorname{NAFLD}(+)(\mathrm{n}=133)$ & $2.04(1.47-2.83)$ & $1.88(1.32-2.67)$ & $2.32(0.81-6.67)$ & $1.67(0.57-4.88)$ \\
\hline
\end{tabular}

Of the 1,523 female patients, 1,263 (82.9\%) had available data on menopausal status.

CRN, colorectal neoplasia; ACRN, advanced colorectal neoplasia; HR, hazard ratio; CI, confidence interval; NAFLD, nonalcoholic fatty liver disease.

${ }^{\mathrm{a}}$ Values were adjusted for NAFLD, age, current or former smoking, family history of colorectal cancer, use of nonsteroidal anti-inflammatory drugs, regular exercise, and baseline adenoma characteristics.

tients, data on menopausal status were available in 1263 (82.9\%). Of these, 863 patients $(68.3 \%)$ were premenopausal and 400 patients (31.7\%) were postmenopausal at the time of the index colonoscopy. NAFLD was associated with an increased risk of metachronous overall CRN in postmenopausal women (adjusted HR, 1.88; 95\% CI, 1.32 to 2.67 ) but not in premenopausal women (adjusted HR, 1.37; 95\% CI, 0.90 to 2.09). NAFLD was not significantly associated with the risk of metachronous ACRN in both premenopausal and postmenopausal women. 


\section{DISCUSSION}

In this large-scale longitudinal study, we found that NAFLD was independently associated with an increased risk of the development of metachronous overall CRN after polypectomy in both men and women. Furthermore, NAFLD was an independent risk factor for metachronous ACRN in women. Our study provides clear evidence on the existence of an association between NAFLD and the development of CRN.

Accumulating epidemiological evidences have demonstrated that patients with NAFLD have a significantly higher prevalence of CRN than those without NAFLD [12-15]. NAFLD is considered a hepatic manifestation of metabolic syndrome $[23,24]$, and is also associated with insulin resistance and a systemic low-grade inflammatory status [25]. NAFLD shares common risk factors with CRN, including central obesity, impaired glucose tolerance, and dyslipidemia $[26,27]$. The most likely potential mechanism linking NAFLD to the development of CRN is the proinflammatory and procarcinogenic effect of insulin resistance and the chronic inflammatory condition of patients with NAFLD $[28,29]$. Elevated serum levels of insulin and insulin-like growth factor may facilitate the development of CRN by promoting the proliferation of colonic epithelial cells and inhibiting apoptosis [27,29,30]. Proinflammatory cytokines, such as tumor necrosis factor- $\alpha$, interleukin (IL)-6, IL-8, and plasminogen activator inhibitor-1, may also play important roles in colorectal carcinogenesis by promoting tumor cell proliferation and angiogenesis, and inhibiting apoptosis [31,32]. Additionally, decreased adiponectin levels and increased leptin levels in patients with NAFLD have procarcinogenic effects on the colorectum [33].

Several studies have reported a significant positive association between NAFLD and the prevalence of CRN [12-15], whereas data on the association between NAFLD and the risk of metachronous CRN at surveillance colonoscopy are extremely limited. To date, only two studies have attempted to investigate this topic $[34,35]$. One retrospective cohort study from Taiwan including 1,522 subjects reported that NAFLD was an independent risk factor for adenoma formation after a negative baseline colonoscopy (adjusted odds ratio [OR], 1.45; 95\% CI, 1.07 to 1.98) [34]. However, the study only investigated the outcome of a specific time point. This is the reason why that study presented OR values from logistic regression analysis rather than HR values from Cox proportional hazards regression analysis. Additionally, in that study, only patients who had no adenoma at the index colonoscopy were included, and the interval between the initial (baseline) colonoscopy and the second colonoscopy (2.59 \pm 1.24 years) was too short to analyze the risk of metachronous adenoma development in patients without adenoma at baseline. Another retrospective study including 441 NAFLD cases and 441 propensity score-matched controls demonstrated that NAFLD independently increased the risk of overall CRN occurrence with marginal significance (adjusted HR, 1.31; 95\% CI, 1.01 to 1.71; $p$ $=0.05$ ) [35]. However, in a subgroup analysis based on index colonoscopy risk categories, the effect of NAFLD on the overall CRN occurrence at the time of surveillance colonoscopy was significant in the normal group (adjusted HR, 1.47; 95\% CI, 1.05 to 2.06; $p=0.02$ ), but not in the low- and high-risk groups. The negative results in the low- and high-risk groups might be because of the small number of patients in these groups. Among 441 subjects with NAFLD, only 85 and 38 were from the low- and high-risk groups, respectively, and among 441 subjects without NAFLD, only 100 and 34 were from the low- and high-risk groups, respectively. Our study with a relatively large sample size (5,176 patients with low-risk adenomas and 1,006 patients with high-risk adenomas) provides reliable evidence on the existence of a positive association between NAFLD and the risk of metachronous CRN in patients with adenoma at the index colonoscopy. Although our study did not include subjects with no adenoma at the index colonoscopy, according to the results of our study and the prior two studies, NAFLD seems to have an influence on metachronous CRN development regardless of the presence or absence of adenoma at the index colonoscopy.

Interestingly, we found that NAFLD was related to an increased risk of metachronous ACRN in women but not in men. This result suggests that NAFLD may have a stronger effect on colorectal carcinogenesis (especially the later stage of the adenoma-carcinoma sequence) in women than in men. The reasons for the sex-associated difference in the impact of NAFLD on the risk of metachronous ACRN development are unclear. Differences in sex hormones between men and women may be one of the reasons for the different results. To better assess the 
effects of sex hormones, we performed a subgroup analysis based on menopausal status. The results showed that NAFLD was associated with an increased risk of metachronous overall CRN in postmenopausal women but not in premenopausal women. These results may indicate that female hormones may have protective effects against metachronous CRN. However, NAFLD was not significantly associated with the risk of metachronous ACRN in postmenopausal women. This insignificant association may be because of the small number of postmenopausal women with metachronous ACRN $(n=7)$. Further studies are warranted to clarify the effects of NAFLD on metachronous CRN according to sex.

The current guidelines recommend the interval of post-polypectomy surveillance based solely on the baseline adenoma characteristics without considering the clinical risk factors for metachronous CRN [3-5]. Given that approximately one-third of the population has NAFLD and the incidence of this condition is markedly increasing [36,37], future risk stratification for surveillance colonoscopy may need to consider clinical risk factors such as NAFLD in addition to baseline adenoma characteristics. This might help provide a more detailed and tailored approach to each individual patient undergoing post-polypectomy surveillance.

To our knowledge, this is the first and largest longitudinal study demonstrating the impact of NAFLD on the risk of metachronous CRN development after adenoma removal. Nevertheless, the current study had several limitations. First, this was a hospital-based study rather than a population-based study and included only patients of Korean ethnicity. Moreover, most subjects were employees of various companies. Therefore, some degree of selection bias was likely present. Second, the possibility of inclusion of missed adenoma from index colonoscopy cannot be ruled out because of the retrospective nature of the study design. However, the likelihood of including missed adenoma is considered to be very low because our study selected and analyzed only high-quality data satisfying colonoscopy quality indicators, such as adenoma detection rate, withdrawal time of $\geq 6$ minutes, and adequate bowel preparation. The overall adenoma detection rate in subjects aged $\geq 50$ years at the index colonoscopy (between 2010 and 2014) was 32\% (39\% for men and $22 \%$ for women), which exceeded the recommended thresholds for colonoscopy quality (i.e., $\geq$
$25 \%$ overall or $\geq 30 \%$ for male patients and $\geq 20 \%$ for female patients) [38]. Third, the interval between the index and follow-up colonoscopy was relatively short (mean, 3.5 years). Accordingly, we did not include subjects without adenoma at the index colonoscopy. Fourth, fatty liver was diagnosed using abdominal US but was not confirmed with histological examination. However, liver biopsy is invasive and unfeasible in outpatients participating in health-checkup programs. Further, hepatic steatosis can be diagnosed using abdominal US with a high accuracy [39]. Lastly, the presence of fatty liver was evaluated only once (at the time of the index colonoscopy). The presence or severity of fatty liver may change over time. We did not assess the effect of dynamic changes of fatty liver status on the risk of metachronous CRN. However, during an average follow-up of 3.5 years, the presence or absence of fatty liver is likely to change in only a very small number of patients.

Despite these limitations, our data provide a better understanding of the association between NAFLD and the risk of metachronous CRN. NAFLD was identified as an independent risk factor for metachronous CRN development in both men and women, and was also an independent risk factor for metachronous ACRN in women. Our results indicate that NAFLD may have an impact on the risk of metachronous CRN development after polypectomy. Further large prospective studies are needed to clarify whether patients with NAFLD need more intensive surveillance after adenoma removal.

\section{KEY MESSAGE}

1. Nonalcoholic fatty liver disease (NAFLD) was independently associated with an increased risk of the development of metachronous overall colorectal neoplasia (CRN) after polypectomy in both men and women.

2. Furthermore, NAFLD was an independent risk factor for metachronous advanced CRN in women.

\section{Conflict of interest}

No potential conflict of interest relevant to this article was reported. 


\section{REFERENCES}

1. Ferlay J, Soerjomataram I, Dikshit R, et al. Cancer incidence and mortality worldwide: sources, methods and major patterns in GLOBOCAN 2012. Int J Cancer 2015;136:Е359-E386.

2. Lin OS, Kozarek RA, Cha JM. Impact of sigmoidoscopy and colonoscopy on colorectal cancer incidence and mortality: an evidence-based review of published prospective and retrospective studies. Intest Res 2014;12:268-274.

3. Lieberman DA, Rex DK, Winawer SJ, Giardiello FM, Johnson DA, Levin TR. Guidelines for colonoscopy surveillance after screening and polypectomy: a consensus update by the US Multi-Society Task Force on Colorectal Cancer. Gastroenterology 2012;143:844-857.

4. Hong SN, Yang DH, Kim YH, et al. Korean guidelines for post-polypectomy colonoscopic surveillance. Korean J Gastroenterol 2012;59:99-117.

5. Cairns SR, Scholefield JH, Steele RJ, et al. Guidelines for colorectal cancer screening and surveillance in moderate and high risk groups (update from 2002). Gut 2010;59:666-689.

6. Kim MC, Jung SW, Kim CS, Chung TH, Yoo CI, Park NH. Metabolic syndrome is associated with increased risk of recurrent colorectal adenomas in Korean men. Int J Obes (Lond) 2012;36:1007-1011.

7. Kim NH, Park JH, Park DI, Sohn CI, Choi K, Jung YS. Metabolic syndrome is a risk factor for adenoma occurrence at surveillance colonoscopy: a single-center experience in Korea. Medicine (Baltimore) 2016;95:e4454.

8. Byrne CD, Targher G. NAFLD: a multisystem disease. J Hepatol 2015;62(1 Suppl):S47-S64.

9. Korean Association for the Study of the Liver (KASL). KASL clinical practice guidelines: management of nonalcoholic fatty liver disease. Clin Mol Hepatol 2013;19:325348.

10. Anstee QM, Targher G, Day CP. Progression of NAFLD to diabetes mellitus, cardiovascular disease or cirrhosis. Nat Rev Gastroenterol Hepatol 2013;10:330-344.

11. Sanna C, Rosso C, Marietti M, Bugianesi E. Non-alcoholic fatty liver disease and extra-hepatic cancers. Int J Mol Sci 2016;17:E717.

12. Lee YI, Lim YS, Park HS. Colorectal neoplasms in relation to non-alcoholic fatty liver disease in Korean women: a retrospective cohort study. J Gastroenterol Hepatol 2012;27:91-95.
13. Stadlmayr A, Aigner E, Steger B, et al. Nonalcoholic fatty liver disease: an independent risk factor for colorectal neoplasia. J Intern Med 2011;270:41-49.

14. Ding W, Fan J, Qin J. Association between nonalcoholic fatty liver disease and colorectal adenoma: a systematic review and meta-analysis. Int J Clin Exp Med 2015;8:322333.

15. Shen H, Lipka S, Kumar A, Mustacchia P. Association between nonalcoholic fatty liver disease and colorectal adenoma: a systemic review and meta-analysis. J Gastrointest Oncol 2014;5:440-446.

16. Ze EY, Kim BJ, Jun DH, Kim JG, Kang H, Lee DY. The Fatty Liver Index: a simple and accurate predictor of colorectal adenoma in an average-risk population. Dis Colon Rectum 2018;61:36-42.

17. Ahn JS, Sinn DH, Min YW, et al. Non-alcoholic fatty liver diseases and risk of colorectal neoplasia. Aliment Pharmacol Ther 2017;45:345-353.

18. Soweid AM, Kobeissy AA, Jamali FR, et al. A randomized single-blind trial of standard diet versus fiber-free diet with polyethylene glycol electrolyte solution for colonoscopy preparation. Endoscopy 2010;42:633-638.

19. Wen CP, David Cheng TY, Tsai SP, et al. Are Asians at greater mortality risks for being overweight than Caucasians?: redefining obesity for Asians. Public Health Nutr 2009;12:497-506.

20. Hamaguchi M, Kojima T, Itoh Y, et al. The severity of ultrasonographic findings in nonalcoholic fatty liver disease reflects the metabolic syndrome and visceral fat accumulation. Am J Gastroenterol 2007;102:2708-2715.

21. Chang Y, Jung HS, Cho J, et al. Metabolically healthy obesity and the development of nonalcoholic fatty liver disease. Am J Gastroenterol 2016;111:1133-1140.

22. Viera AJ, Garrett JM. Understanding interobserver agreement: the kappa statistic. Fam Med 2005;37:360-363.

23. Marchesini G, Brizi M, Bianchi G, et al. Nonalcoholic fatty liver disease: a feature of the metabolic syndrome. Diabetes 2001;50:1844-1850.

24. Williams CD, Stengel J, Asike MI, et al. Prevalence of nonalcoholic fatty liver disease and nonalcoholic steatohepatitis among a largely middle-aged population utilizing ultrasound and liver biopsy: a prospective study. Gastroenterology 2011;140:124-131.

25. Targher G, Bertolini L, Scala L, Zoppini G, Zenari L, Falezza G. Non-alcoholic hepatic steatosis and its relation to increased plasma biomarkers of inflammation and 
endothelial dysfunction in non-diabetic men: role of visceral adipose tissue. Diabet Med 2005;22:1354-1358.

26. Ahmed RL, Schmitz KH, Anderson KE, Rosamond WD, Folsom AR. The metabolic syndrome and risk of incident colorectal cancer. Cancer 2006;107:28-36.

27. Giovannucci E. Metabolic syndrome, hyperinsulinemia, and colon cancer: a review. Am J Clin Nutr 2007;86:s836-s842.

28. Sanyal AJ, Campbell-Sargent C, Mirshahi F, et al. Nonalcoholic steatohepatitis: association of insulin resistance and mitochondrial abnormalities. Gastroenterology 2001;120:1183-1192.

29. Wong VW, Hui AY, Tsang SW, et al. Metabolic and adipokine profile of Chinese patients with nonalcoholic fatty liver disease. Clin Gastroenterol Hepatol 2006;4:1154-1161.

30. Renehan AG, Zwahlen M, Minder C, O'Dwyer ST, Shalet SM, Egger M. Insulin-like growth factor (IGF)-I, IGF binding protein-3, and cancer risk: systematic review and meta-regression analysis. Lancet 2004;363:1346-1353.

31. Kim S, Keku TO, Martin C, et al. Circulating levels of inflammatory cytokines and risk of colorectal adenomas. Cancer Res 2008;68:323-328.

32. Mutoh M, Niho N, Komiya M, et al. Plasminogen activator inhibitor-1 (Pai-1) blockers suppress intestinal polyp formation in Min mice. Carcinogenesis 2008;29:824-829.

33. Yamaji T, Iwasaki M, Sasazuki S, Tsugane S. Interaction between adiponectin and leptin influences the risk of colorectal adenoma. Cancer Res 2010;70:5430-5437.

34. Huang KW, Leu HB, Wang YJ, et al. Patients with n onalcoholic fatty liver disease have higher risk of colorectal adenoma after negative baseline colonoscopy. Colorectal Dis 2013;15:830-835.

35. Yang YJ, Bang CS, Shin SP, Baik GH. Clinical impact of non-alcoholic fatty liver disease on the occurrence of colorectal neoplasm: propensity score matching analysis. PLoS One 2017;12:e0182014.

36. Mikolasevic I, Orlic L, Stimac D, Hrstic I, Jakopcic I, Milic S. Non-alcoholic fatty liver disease and colorectal cancer. Postgrad Med J 2017;93:153-158.

37. Younossi ZM, Koenig AB, Abdelatif D, Fazel Y, Henry L, Wymer M. Global epidemiology of nonalcoholic fatty liver disease: meta-analytic assessment of prevalence, incidence, and outcomes. Hepatology 2016;64:73-84.

38. Rex DK, Boland CR, Dominitz JA, et al. Colorectal cancer screening: recommendations for physicians and patients from the U.S. Multi-Society Task Force on Colorectal Cancer. Am J Gastroenterol 2017;112:1016-1030.

39. Hernaez R, Lazo M, Bonekamp S, et al. Diagnostic accuracy and reliability of ultrasonography for the detection of fatty liver: a meta-analysis. Hepatology 2011;54:1082-1090. 